\title{
Behaviour of Ti-doped 3D Carbon Fibre Composites under Intense Thermal Shock Tests
}

A.Centeno ${ }^{\mathrm{a}}$, C. Blanco ${ }^{\mathrm{a} *}$, R. Santamaría ${ }^{\mathrm{a}}, \mathrm{M}$. Granda $^{\mathrm{a}}$, R. Menéndez ${ }^{\mathrm{a}}$, G. Pintsuk ${ }^{\mathrm{b}}$ and J. Linke ${ }^{\mathrm{b}}$

${ }^{a}$ Instituto Nacional del Carbón (CSIC), Apdo. 73, 33080, Oviedo, Spain

${ }^{b}$ Forschungszentrum Jülich, EURATOM Association, 52425, Jülich, Germany

PACS Number: 28.52.Fa

\begin{abstract}
.-
This paper reports on the development of novel Ti-doped 3D carbon fibre composites (CFCs) and their performance when exposed to transient thermal loads (disruptions) in the electron beam facility JUDITH at different conditions. Depending on the applied load, the CFCs showed three steps of erosion: 1) breaking of PAN fibres with pull out from the surface; 2) cracking and ablation of pitch fibres close to the interface of PAN/pitch fibre-bundles; 3) finally, erosion of pitch fibres in the centre of the bundle. The addition of titanium carbide resulted in a significant improvement in thermal shock behaviour of these materials compared to their undoped counterparts.
\end{abstract}

* C. Blanco: corresponding author; clara@incar.csic.es; Tel.: +34 985118994 Fax.: +34 985297662 


\section{INTRODUCTION}

In the future tokamak ITER, carbon fibre composites with a three directional (3D) structure are considered as plasma-facing materials (PFMs) for the vertical targets in the divertor, the part exposed to the most severe conditions, due to their favorable thermo-mechanical properties [1]. During normal operation design heat load up to 20 $\mathrm{MW} / \mathrm{m}^{2}$ will be deposited and during off-normal events the plasma facing materials will be exposed to transient heat fluxes of several $\mathrm{GW} / \mathrm{m}^{2}$ in the range of ms [2]. An evaluation of the erosion lifetime of CFCs showed that it is affected by physical sputtering and chemical erosion during the normal operations. Additionally during transient thermal loads such as disruptions, plasma vertical displacements events (VDEs) and edge localized modes (ELMs), carbon materials suffer from sublimation, crack formation and macroscopic erosion (brittle destruction) and from dust formation $[3,4]$. Therefore, high thermal shock resistance is required for protective materials to guarantee the lifetime of components. The eroded carbon atoms are redeposited at the walls of the vacuum vessel and have high affinity for the formation of tritiated hydrocarbons causing safety problems [5].

In order to improve the thermal shock resistance of CFCs several studies based on experiments and numerical simulation data showed the importance of the selection of an optimum fibre orientation. Fibres parallel to the loaded area have to be avoided as much as possible to limit the preferential erosion of CFCs under transient thermal shock loads [6-8]. The development of CFCs with different dopants such as carbides of $\mathrm{Ti}, \mathrm{W}$ or $\mathrm{Zr}$ is one of the possibilities to improve some deficient properties of these materials. These carbides have a catalytic effect on graphitization of carbon and, consequently, contribute to improve the thermal conductivity [9] and therefore their 
thermal shock resistance. Additionally, these elements also contribute to improve the resistance to chemical erosion [10].

This paper studies the development of novel Ti-doped CFCs with two different 3D carbon fibre preforms and their erosion mechanisms when exposed to disruptions in the electron beam facility JUDITH.

\section{EXPERIMENTAL}

\subsection{Manufacturing of 3D CFCs}

Four CFCs (two undoped materials and two Ti-doped ones) were developed using two different 3D carbon fibre preforms as reinforcement (CF1 and CF2), supplied by SGL Carbon Group. A commercial mesophase pitch (AR) supplied by Mitsubishi Gas Chemical was used as matrix precursor. TiC nanoparticles $(80 \mathrm{~nm})$ were used as dopant. Densification was performed by liquid impregnation with the matrix precursor, either the mesophase pitch or the doped matrix precursor, following similar procedure to that described in [11]. The resultant materials were carbonised at $1000^{\circ} \mathrm{C}$. After several densification cycles, materials were graphitised at $2700^{\circ} \mathrm{C}$. The undoped materials were labelled as U-CF1 and U-CF2 and the Ti-doped materials as Ti-CF1 and Ti-CF2.

\subsection{Characterisation of CFCs}

Materials developed were characterised by measuring their bulk density, open porosity by Archimedes water-immersion and close porosity was calculated from the difference in real density of the bulk material and that obtained after grinding $(<75 \mu \mathrm{m})$, determined by helium pycnometry. 
Their microstructure was studied by SEM. The thermal conductivity at room temperature was calculated from the thermal diffusivity measured in the x-direction with a laser flash apparatus, the bulk density and the specific heat.

\subsection{Thermal shock tests}

Thermal shock tests were performed in the electron beam test facility JUDITH [12] on specimens of $12 \times 12 \times 5 \mathrm{~mm}^{3}$ where pitch fibre-bundles were aligned parallel to the heat transfer direction, while PAN fibre-bundles were aligned perpendicular to it. Absorbed power densities of $1,1.6$ and $2.4 \mathrm{GW} / \mathrm{m}^{2}$ with a pulse duration of $5 \mathrm{~ms}$ were applied at room temperature on an area of $4 \times 4 \mathrm{~mm}^{2}$. Multiple shots were performed (10 and 100 shots) to investigate the evolution of the damage caused in the materials. For diagnostics during the experiments, a CCD-camera was used to observe particle release during the thermal loads [13]. By the current measurement the absorbed power density, the exact pulse duration and hence the absorbed energy density was determined. A fast pyrometer was used to measure the surface temperature during the shots. After the tests, the erosion depth in the materials was characterised using laser profilometry and observing the cross section of the loaded area. SEM and optical microscopy were used to study the microstructures of the materials.

\section{RESULTS AND DISCUSSIONS}

\subsection{Characterisation of the CFCs}

The carbon fibre preforms used (CF1 and CF2) contain pitch fibre-bundles in the xdirection and PAN carbon fibre-bundles in the y and z-directions. The structure of 
CF1 is more anisotropic than CF2. While CF1 has the three different planes, CF2 has the typical orthogonal structure where XY and YZ-planes are equivalent. Besides, CF1 is constituted by two different fibre-layers in each direction making the structure more complex. Both preforms have 40 vol. \% porosity. The percentage of fibres in each direction for CF1 is $33 \%$ of pitch fibres in the x-direction and $15 \%$ and $12 \%$ of PAN fibres in the y and z-directions respectively. The total amount of fibres in CF2 is the same, although the proportion in each direction varies: $43 \%$ of pitch fibres and $8 \%$ and $9 \%$ of PAN fibres. In both performs large pores (>1mm) are found at regular positions as a consequence of the intersection of fibre-bundles, which appear filled of matrix after the densification step. In the doped materials, TiC is well dispersed in the matrix in sub-micrometric size. The porosity is entirely open, with values of 20 vol. \% for materials developed with CF1 and 17 vol. \% for those with CF2, and bulk densities of $\sim 1.50 \mathrm{~g} / \mathrm{cm}^{3}$ and $\sim 1.70 \mathrm{~g} / \mathrm{cm}^{3}$ respectively. The values of thermal conductivity at room temperature were $179 \mathrm{~W} / \mathrm{mK}$ and $186 \mathrm{~W} / \mathrm{mK}$ for U-CF1 and Ti-CF1 respectively, while materials developed with the orthogonal preform showed significantly higher thermal conductivity $(220 \mathrm{~W} / \mathrm{mK}$ for U-CF2 and $288 \mathrm{~W} / \mathrm{mK}$ for Ti-CF2). Ti-CF2 showed the highest value due to the high catalytic effect of titanium on graphitisation.

\subsection{Response of materials to transient heat loads}

The current measurements (Figure 1) showed some particularities which help to establish differences between the behaviour of the materials studied. In an ideal behaviour (without erosion) all the current applied should be absorbed by the 
material. However, in the materials tested, an early attenuation is observed in the first shot, which is related to macroscopic erosion (brittle destruction) [3, 4]. The CCD camera showed large rays of light corresponding to the release of hot particles originating from PAN fibres aligned perpendicular to the heat conduction direction. Fibres broke as a consequence of the induced thermal stresses caused by great thermal gradients and also because of the anisotropy of the CFCs [8]. With increasing the number of shots the attenuation of the measured current occurs later in time. This could originate from the geometrical modification of the surface and the related reduction of thermally induced stresses at the surface which cause the erosion of macroscopic parts of the PAN fibres during the first shots. Therefore, the mechanical stresses will tend to equilibrate with the increasing number of shots being dominant the thermal erosion. This was also reflected in the particle emission images, where the number of ejected particles decreased significantly. At $1 \mathrm{GW} / \mathrm{m}^{2}$, the evolution of the current is rather similar for all the materials, being clearly improved as the signal attenuation is negligible after 100 shots. Differences start to become significant at higher absorbed power densities $\left(1.6 \mathrm{GW} / \mathrm{m}^{2}\right)$. The differences between U-CF1 and U-CF2 (Figure 1 (a) and (b)) are especially notable at 100 shots, as in U-CF2 the attenuation of the measured current is clearly delayed. Significant differences were also observed in surface temperature evolution. U-CF2 reached lower surface temperature (Figure 1 (b)) as a direct consequence of its higher thermal conductivity and, therefore, better heat transfer. On the other hand, the surface temperature decreased with increasing number of shots for all materials. This is related to the reduction of PAN fibres-bundle area where was overheated during shots due to the poor heat removal into the bulk. This decrease is not so significant 
for U-CF2, probably due to a smaller amount of eroded PAN fibres. For Ti-doped materials, the attenuation of the measured current at $1.6 \mathrm{GW} / \mathrm{m}^{2}$ after 100 shots occurs later indicating that the erosion is lower. The evolution for Ti-CF1 at 2.4 $\mathrm{GW} / \mathrm{m}^{2}$ is rather similar to that for $\mathrm{U}-\mathrm{CF} 1$ at $1.6 \mathrm{GW} / \mathrm{m}^{2}$, as can be observed in the curves in Figure 1 (a) and (c), showing the better thermal response of the doped material. The evolution of the current curve of Ti-CF2 together with the evolution of temperature indicates a better thermal response than for Ti-CF1 (see curves (c) and (d)), as a consequence of its higher thermal conductivity.

\subsection{Study of the damage produced in the CFCs}

A detailed study of the loaded surfaces revealed the different erosion mechanisms that took place in the CFCs. Depending on the applied load and the characteristics of the material, three steps of erosion were found: 1) breaking of PAN fibres and pull out from the loaded surface; 2) cracking and ablation of pitch fibres close to the interface of PAN/pitch fibre-bundles; 3) erosion of pitch fibres in the centre of the bundle. In general, for all the materials tested under the applied conditions, the loaded area is characterised by a preferential erosion of the PAN fibre-bundles due to their low thermal conductivity perpendicular to the fibre-bundle orientation and the only possibility of heat removal in the lateral direction. This is observed for both carbon fibre preforms, images in Figure 2 (a) and (b). Besides, a lower erosion depth can be observed in the material produced with the preform CF2. However the values obtained for the erosion depth of PAN fibre-bundles should not be taken as absolute numbers due to the large scattering $(\sim \pm 100 \mu \mathrm{m})$ caused by the complex structure of the materials and the presence of porosity. Nonetheless, the values confirm that the 
erosion rate of PAN fibre-bundles decreased significantly after 100 shots concluding that the first shots have the strongest impact on the material ( $60 \%$ related to 100 applied shots). The erosion in the doped materials seems to be slightly lower ( 10\%) than for the undoped ones and, as mentioned before, the erosion depth of the materials developed with CF2 was significantly lower (e.g. 650 $\mu \mathrm{m}$ vs $\sim 1000 \mu \mathrm{m}$ after 100 shots at $2.4 \mathrm{GW} / \mathrm{m}^{2}$ ).

Pitch fibres close to the interface between PAN and pitch fibre-bundles appeared ablated (with conical shape typical for sublimation) due to the contact with overheated PAN fibres. This happened in all materials especially at power densities higher than $1 \mathrm{GW} / \mathrm{m}^{2}$. Nonetheless, the doped materials showed higher resistance. Figure 2 (c) and (d) show the microstructure of the pitch fibres for U-CF2 and TiCF2. For the latter, erosion of pitch fibres took place mainly until a distance of $\sim 60$ $\mu \mathrm{m}$ from the interface, while for U-CF2 high amounts of eroded pitch fibres were found up to a distance of $\sim 120 \mu \mathrm{m}$.

At the centre of pitch fibre-bundle, erosion was observed for U-CF1 at $1.6 \mathrm{GW} / \mathrm{m}^{2}$ after 100 shots. At this power density, in Ti-CF1 the pitch fibres remained almost undamaged and only become more affected at $2.4 \mathrm{GW} / \mathrm{m}^{2}$. This is related to the drop detected in the absorbed current curve at the $100^{\text {th }}$ shot (Figure 1 (a) and (c)). Materials developed with CF2 again showed a better behaviour than those obtained with CF1 and the doped one showed a better thermal shock resistance than the undoped one. A combination of the higher thermal conductivity and the agglomeration of TiC on the surface, mainly in the transition zone between PAN and pitch fibre-bundles, might have a shielding effect on pitch fibres in the centre of the bundle resulting in less erosion compared with undoped CFCs. Figure 2 (e) and (f) 
show details of the microstructure of fibres in the centre of the bundle for U-CF2 and Ti-CF2, respectively, outlining the higher resistance of the doped material.

Besides erosion, also crack formation plays an important role. These occurred mainly close to the interface, either along the fibre axis (Figure 3 (a)) or across to the fibrebundles (Figure 3 (b)). Cracks along the fibre axis appear during loading due to the shrinkage along their longitudinal direction and expansion in their transverse one. The erosion in pitch fibres starts with the formation of cracks along the fibre axis and with increasing power density and temperature sublimation is observed. Especially at $2.4 \mathrm{GW} / \mathrm{m}^{2}$ in the undoped materials, where the erosion was quite high, most of the fibres showed conical shape typical for sublimation, while in doped materials a higher number of these cracks and less sublimation was found because of their higher thermal conductivity and the therefore higher thermal shock resistance. Also differences between thermal coefficient expansions could be responsible in the formation of cracks. On the cross sections analysed by optical microscopy (Figure 3 (b)), horizontally aligned cracks were found in pitch fibre-bundles in the materials developed with CF2. For CF1 such cracks were not observed because of sublimation of pitch fibres took place causing the erosion down to the depth of horizontal crack formation. For the used electron beam $(\mathrm{U}=120 \mathrm{keV})$ the penetration depth is about 60-70 $\mu \mathrm{m}$ and therefore larger than the depth in which the cracks in the pitch fibres were observed ( $\sim 30-50 \mu \mathrm{m}$ from the surface). The presence of these cracks is in agreement with the results obtained for the reference CFC, i.e. NB31, tested in a Quasi-Stationary Plasma Accelerator [14] after ELM-like loading where the penetration depth is negligible. Therefore with a very high probability the influence 
of the penetration depth of the electron beam can be excluded and it is just a matter of thermally induced stresses.

\section{CONCLUSIONS}

Three steps of erosion were identify for the materials studied depending on the applied loads: 1) breaking of PAN fibres with pull out from the surface; 2) cracking and ablation of pitch fibres close to the interface of PAN/pitch fibre-bundles; 3) finally, erosion of pitch fibres in the centre of the bundle. Materials developed with the orthogonal framework showed better a thermal response under transient thermal loads due to their higher thermal conductivity and also due to their more isotropic structure. The addition of titanium carbide resulted in a significant improvement in thermal shock behaviour of these materials compared to their undoped counterparts due to the higher thermal conductivity resultant from the catalytic effect of titanium on graphitisation.

\section{ACKNOWLEDGEMENTS}

The authors would like to thank SGL Carbon Group for supplying the 3D preforms. This work has been performed within the framework of the Integrated European Project 'ExtreMat' (contract NMP-CT-2004-500253) with financial support by the European Community.

\section{REFERENCES}

[1] M. Merola et al 2004 Phys. Scr. T111152

[2] G. Federici 2009 J. Nucl. Mater. 386-388 720 
[3] Y. Koza et al 2004 J. Nucl. Mater. 329-333 706

[4] J. Linke et al 2001 J. Nucl. Mater. 290-293 1102

[5] H. Yoshida et al 2004 Fusion Eng. Des. 70201

[6] J. Compan et al 2007 Phys.Scr. T 128246

[7] J. Compan et al 2009 J. Nucl. Mater. 386-388 797

[8] S. Pestchanyi and I. Landman 2006 Fusion Eng. Des. 81275

[9] I. López-Galilea et al 2007 Phys.Scr. T 12860

[10] M. Balden et al 2001 J. Nucl. Mater. 290-293 52

[11] A. Centeno et al 2009 J. Mater. Sci. 442525

[12] T.Hirai et al 2005 Mater.Trans 46412

[13] T.Hirai et al 2003 J.Nucl.Mater. 321110

[14] K. Wittlich et al 2009 Fusion Eng. Des. 7-11 1982 


\section{Figure Captions}

Figure 1.- Evolution of absorbed current and surface temperature versus time during $t_{\text {pulse }}=5$ ms and multiple shots: (a) U-CF1, $1.6 \mathrm{GW} / \mathrm{m}^{2}$ (b) U-CF2, $1.6 \mathrm{GW} / \mathrm{m}^{2}$, (c) TiCF1, $2.4 \mathrm{GW} / \mathrm{m}^{2}$ and (d) Ti-CF2, $2.4 \mathrm{GW} / \mathrm{m}^{2}$

Figure 2.- $\mathrm{SEM}$ micrographs after testing at $\mathrm{P}_{\mathrm{abs}}=1.6 \mathrm{GW} / \mathrm{m}^{2}, \mathrm{t}_{\text {pulse }}=5 \mathrm{~ms}$ and 100 shots (a) Ti-CF1; (b) Ti-CF2, (c) Ti-CF2, (d) U-CF2,(e) Ti-CF2 and (f) U-CF2

Figure 3.- Cracking of pitch fibre-bundles of Ti-CF2 at $\mathrm{P}_{\mathrm{abs}}=2.4 \mathrm{GW} / \mathrm{m}^{2}, \mathrm{t}_{\text {pulse }}=5 \mathrm{~ms}$ and 100 shots: (a) along fibre axis (b) across pitch fibre-bundle 

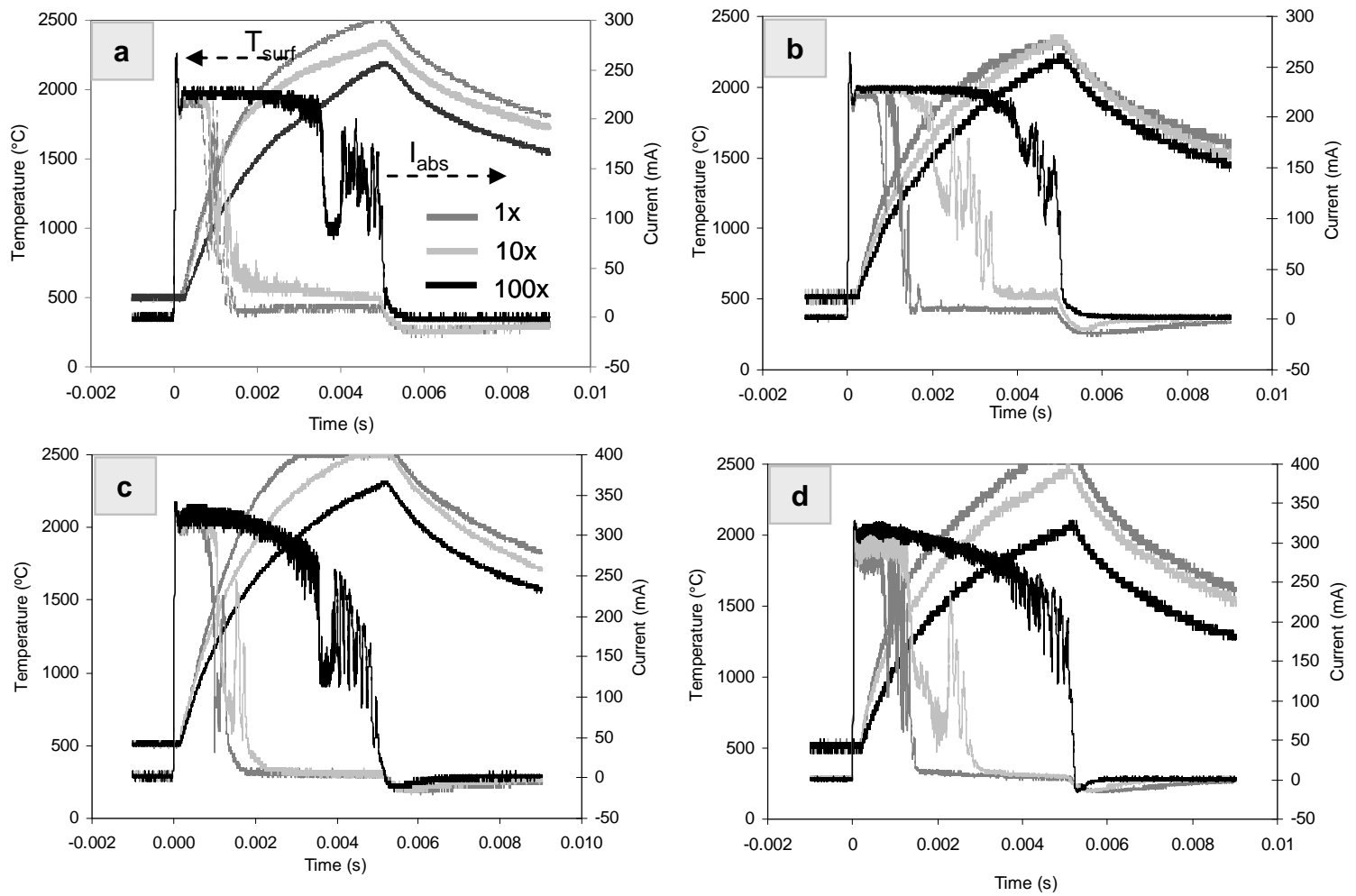

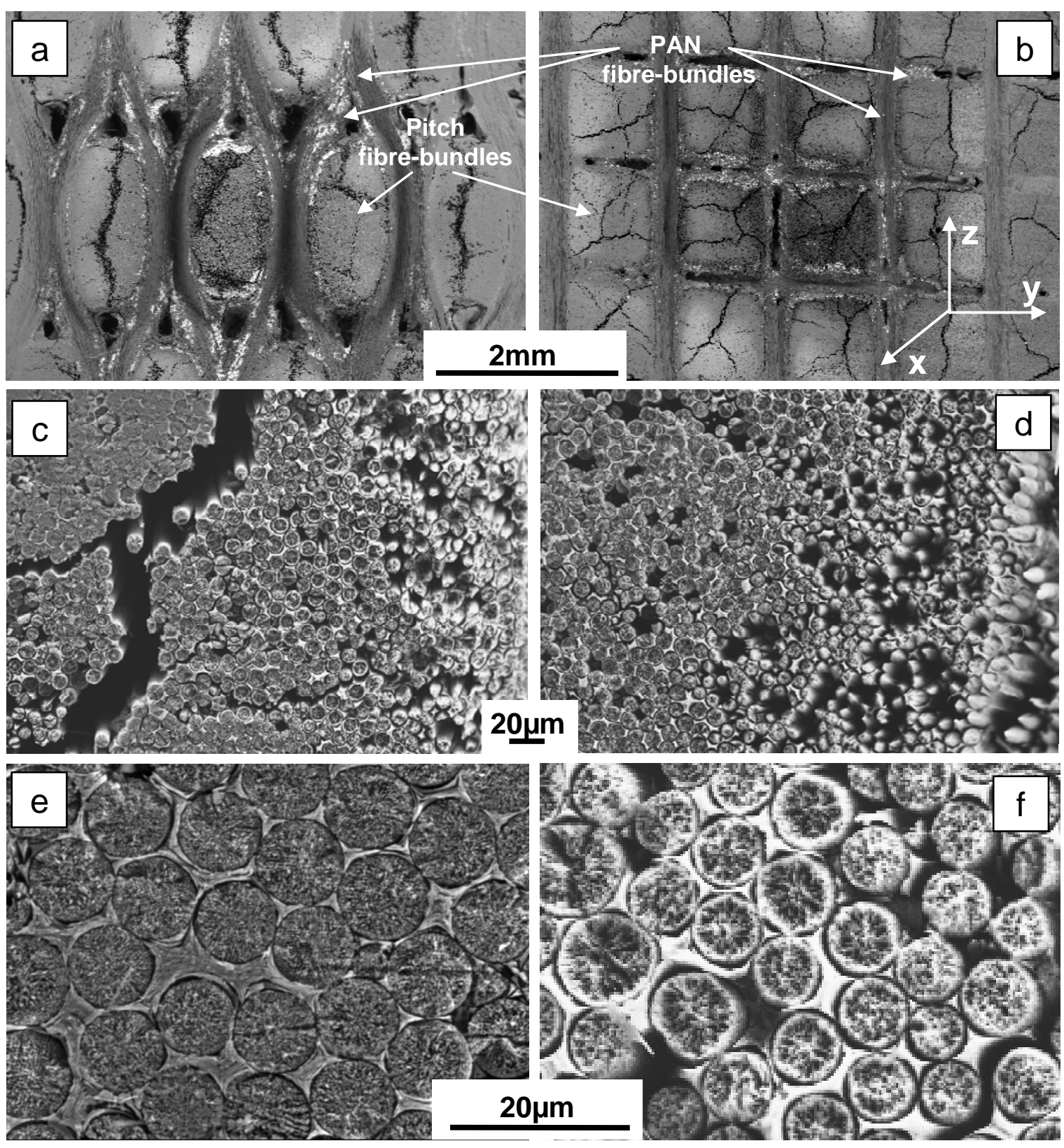


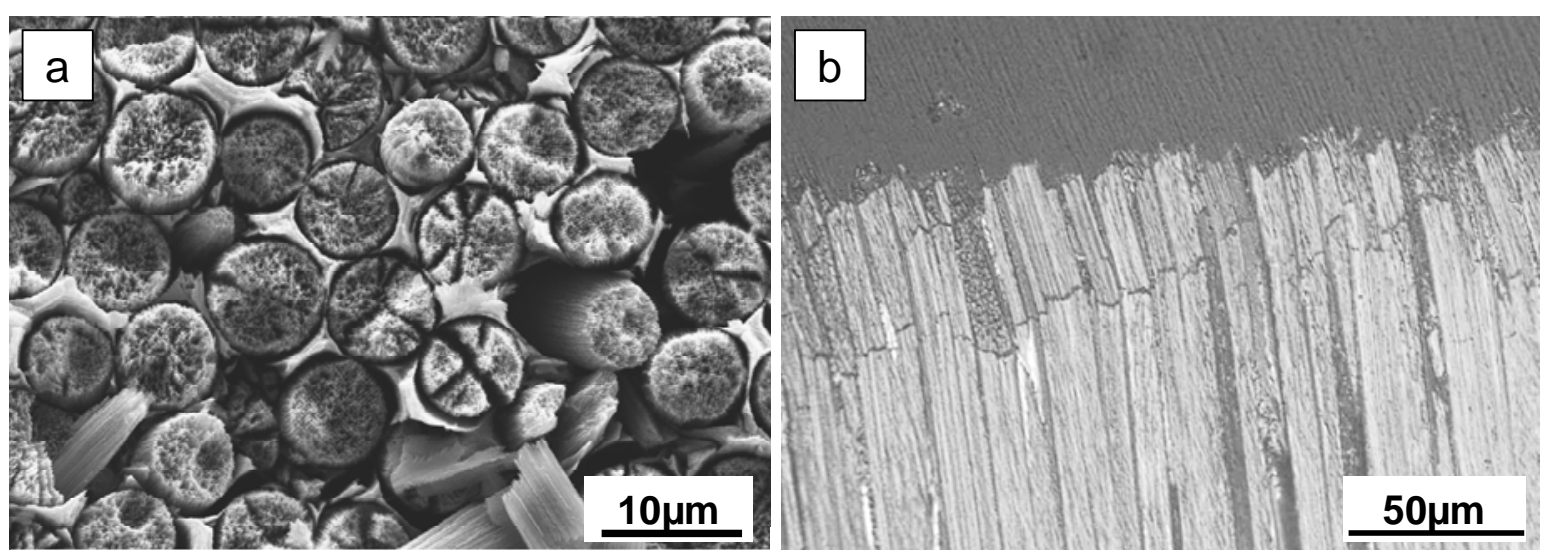

